\title{
Evaluation of Domain Modeling Decisions for Two Identical Domain Specific Languages
}

\author{
Christopher Preschern, Nermin Kajtazovic, and Christian Kreiner
}

\begin{abstract}
Decisions on domain model representations and on domain model scoping are a key task when developing software product lines. However, there is little guidance for $\mathbf{t}$ he choice whether a domain model should be represented by feature-oriented domain models or by domain-specific models and whether a domain should be described by a single domain model or by multiple domain models.

In this paper we apply a method for assessing domain models on two automation system case studies which use domain specific languages (DSLs). The two case studies cover the same domain (warehouse automation) and were developed independently from one another. We evaluate how well the results of the domain model assessment method are suited for our case studies. For the evaluation we consider the development times of the DSLs, the size of the models, and we calculate DSL and code complexity metrics.
\end{abstract}

Index Terms-Automation systems, domain engineering, domain-specific languages.

\section{INTRODUCTION}

Due to the increasing complexity of automation software, domain-specific modeling becomes more and more relevant in this sector. The architecture of the underlying domain model is a crucial design decision which has to be made rather early during system design when usually not much information about the specific domain is available.

In this paper we validate a method (called MADMAPS [1]) for evaluating the feasibility of domain models. We apply the method to two warehouse automation system DSL case studies. The two DSLs cover the same domain and were developed from the same requirements. One of the DSLs adheres to the MADMAPS design suggestion and the other DSL does not. In this paper we want to investigate whether the domain model representation suggested by MADMAPS is better in terms of development time, DSL and code complexity metrics, and modeling effort. These metrics can very well be compared for the two DSLs, because they cover the same domain and the same models were built with both DSLs.

\section{Domain Model Evaluation Method}

In this section we explain a method which we use to evaluate the suitability of the domain models of our

Manuscript received July 15, 2013; revised August 28, 2013

Christopher Preschern, Nermin Kajtazovic, and Christian Kreiner are with the Institute for Technical Informatics, Graz University of Technology, Austria (e-mail: christopher.preschern@tugraz.at, nermin.kajtazovic@ tugraz.at, christian.kreiner@tugraz.at). automation system DSL case studies.

TABLE I: MADMAPS ASSESSMENT CRITERIA

\begin{tabular}{|l|l|}
\hline \hline $\begin{array}{l}\text { Assessment } \\
\text { criteria }\end{array}$ & Description \\
\hline $\begin{array}{l}\text { Fixed relation } \\
\text { Variable relation }\end{array}$ & $\begin{array}{l}\text { In general, domain models consist of elements } \\
\text { and relations. Fixed relations do not vary } \\
\text { between different products in the domain and } \\
\text { variable elements may change. This criteria } \\
\text { expresses the ratio between fixed and variable } \\
\text { relations. }\end{array}$ \\
\hline $\begin{array}{l}\text { Several instances } \\
\text { of elements }\end{array}$ & $\begin{array}{l}\text { This criteria tells whether there should be several } \\
\text { instances of domain model elements. This is } \\
\text { usually the case if real world objects described by } \\
\text { the domain model are instantiated. }\end{array}$ \\
\hline $\begin{array}{l}\text { Different binding } \\
\text { times/views }\end{array}$ & $\begin{array}{l}\text { This criteria rates whether the domain model } \\
\text { should represent different binding times } \\
\text { (compile-time, load-time, runtime, ...) or } \\
\text { different views (abstraction levels). }\end{array}$ \\
\hline $\begin{array}{l}\text { Domain model } \\
\text { used by } \\
\text { non-expert }\end{array}$ & $\begin{array}{l}\text { This criteria rates whether the domain model will } \\
\text { just be used by domain experts or also by end } \\
\text { users. }\end{array}$ \\
\hline \hline
\end{tabular}

TABLE II: MADMAPS ASSESSMENT CRITERIA WEIGHTS [1]

\begin{tabular}{|l|c|c|}
\hline \hline Assessment criteria & $\begin{array}{c}\text { Domain-specifi } \\
\text { c modeling }\end{array}$ & $\begin{array}{c}\text { Feature-orient } \\
\text { ed domain } \\
\text { modeling }\end{array}$ \\
\hline $\begin{array}{l}\text { Fixed relation } \geq \text { Variable } \\
\text { relation }\end{array}$ & 4 & 17 \\
\hline Several instances of elements & 31 & 4 \\
\hline Different binding times/views & 8 & 15 \\
\hline $\begin{array}{l}\text { Domain model used by } \\
\text { non-expert }\end{array}$ & 8 & 15 \\
\hline \hline
\end{tabular}

MADMAPS (multi-attribute domain modeling approach for paradigm selection) [1] is a method to evaluate specific domains in order to decide which domain modeling approach - domain specific modeling (DSM) or feature oriented domain modeling (FODM) - is more suitable for the domain. MADMAPS is based on multi-attribute utility theory [2]. One has to rate assessment criteria for the target domain based on which MADMAPS either recommends to use a domain-specific model, a feature-oriented domain model, or to split the domain up and decide for each of the subdomains separately. Table I presents the assessment criteria which were gathered in [1] based on industrial experience. For more detailed explanations about the criteria and why these criteria were chosen see [1].

To evaluate a specific domain, for each of these criteria one has to answer whether he strongly disagrees $(-2)$, disagrees (-1), neither disagrees nor agrees (0), agrees (1), or strongly agrees (2). These values are weighted with the weighing factors given in Table II. The weighed values are 
summed up for domain-specific modeling and for feature oriented modeling. If one of the two values is significantly higher, then MADMAPS suggests to use that approach. If the values are rather close, MADMAPS suggests to split up the domain model into subdomains and to apply MADMAPS to both subdomains.

TABLE III: MADMAPS CRITERIA WEIGHTS FOR THE WAREHOUSE AUTOMATION DOMAIN

\begin{tabular}{|c|c|c|c|c|c|c|c|c|c|}
\hline \multirow{2}{*}{ Assessment criteria } & \multicolumn{3}{|c|}{ Combined View } & \multicolumn{3}{|c|}{ Combined View } & \multirow[b]{2}{*}{ Rating } & \multirow[b]{2}{*}{ DSM } & \multirow[b]{2}{*}{ FODM } \\
\hline & Rating & DSM & FODM & Rating & DSM & FODM & & & \\
\hline Fixed relation $\geq$ Variable & -1 & 4 & 17 & -1 & 4 & 17 & 17 & 4 & 17 \\
\hline Several instances of elements & 1 & 31 & 4 & 1 & 31 & 4 & 4 & 31 & 4 \\
\hline Different binding times/views & 2 & 8 & 15 & -2 & 8 & 15 & 15 & 8 & 15 \\
\hline Domain model used by & 2 & 8 & 15 & 2 & 8 & 15 & 15 & 8 & 15 \\
\hline$S U M$ & & 59 & 47 & & 27 & -13 & & 3 & -58 \\
\hline
\end{tabular}

\section{CASE STUdies}

In this section we present two DSL case studies and explain their underlying domain model architecture. Both case studies each cover the warehouse automation domain.

Both of the two case studies were carried out as student projects at Graz University of Technology. Both students were given the same requirements and the same information about the warehouse automation domain, as well as a specific hardware prototype of the warehouse automation system (see Fig. 1).

The warehouse automation system consists of conveyor belts, rotary tables, cranes, and a high-bay rack. Each of these elements is equipped with a sensor which detects if packets are currently lying on the element. Every element can be steered independently from the others. The warehouse automation system has to be able to deliver packets between any arbitrary elements within the system. Commands for the delivery are given manually. The system has to detect if other packets block the way and appropriate countermeasures have to be taken (e.g. waiting until the path is free or re-routing the packet if redundant paths are available).

The students had to develop a product line for warehouse automation systems. The amount and position of the different elements should be flexible. For example, with the product line it should be easy to develop a new warehouse automation system which differs in the conveyor belt positions compared to system presented in Fig. 1. The students were given the requirements that different warehouse systems can easily be modeled and that the full automation software including the IEC 61131-3 source code and the automation system visualization should be generated from these models.

\section{A. Warehouse Automation System Product Line-1}

We furthermore call the first case study Warehouse Automation System Product Line - 1 (WASPL-1). WASPL-1 uses domain-specific modeling to describe the warehouse automation system. A DSL is constructed with the DSL tool MetaEdit+. From the DSL model, the source files for the automation program as well as a system visualization are automatically generated. One major challenge for the domain model is that is has to include the logical view (positions of elements like conveyor belts and their connections) as well as the physical view (hardware mapping of sensors/actuators to the automation unit). WASPL-1 solves this problem by integrating both views into a single DSL. The hardware mapping is realized as properties of the basic DSL objects (e.g. conveyor belts). Fig. 2 shows an example model of WASPL-1, where the hardware mapping is textually configured for each DSL element.

\section{B. Warehouse Automation System Product Line-2}

We furthermore call the second case study Warehouse Automation System Product Line - 2 (WASPL-2). WASPL-2 also uses the MetaEdit+ tool to construct a DSL for warehouse automation systems. However, one major difference to WASPL-1 is that WASPL-2 uses a different approach to address the concern of having to model both, the logical and the physical view. WASPL-2 has got a clear separation between the logical and the physical view in the models. Logical elements such as conveyor belts are modeled similar to WASPL-1. Physical elements (automation input/output units and the wires connected to these units) are modeled in a separate diagram. In the whole DSL design these two things are strictly kept apart (DSL relations and code generators in one diagram do not depend on relations and code generators relevant for the other diagram). The two diagrams are strictly separated and could also be represented as two different DSLs. However, with the MetaEdit+ tool is is easier to still treat them as a single DSL and therefore the physical and the logical view are still realized as a single DSL in the tool. Fig. 3 shows an example model of WASPL-2.

\section{Evaluation}

In this section we apply the MADMAPS approach to the two DSLs and furthermore evaluate whether the MADMAPS domain model suggestions are well suited for the warehouse automation domain. For this evaluation we compare several metrics for the two DSLs.

\section{A. MADMAPS}

To apply MADMAPS, we first have to rate the MADMAPS assessment criteria for the warehouse automation domain. The left-hand side (column Combined view) of Table III shows our evaluation for the overall warehouse automation domain. We can see that the results for the DSM and FODM values are very close together and do not significantly favour one method over the other. The MADMAPS approach suggests in such a case to divide the domain into subdomains and evaluate each of the subdomains separately. We split the warehouse automation domain into the Logical view (e.g. conveyor belt arrangement) and the Physical view (automation hardware, wire 
connections). With this separation, the value for the third assessment criteria (different binding times/views) is affected. Also the domain model used by non-expert criteria is affected, because for the warehouse automation case studies it should be possible that a non-expert (e.g. warehouse owner) is able to model the arrangement of elements (e.g conveyor belts) in a system. If the domain model is split into the logical and the physical view, then it would be possible that the warehouse owner models the logical view, while a automation domain expert models the corresponding physical view. Therefore the ratings in Table III strongly differ for the last criteria.

The evaluation results of the subdomains show a more significant separation between the DSM and FODM values. According to the MADMAPS approach this means that for the warehouse automation system it is better to split the domain model into these two subdomains. Both subdomains show a higher ranking for the DSM approach. This means that MADMAPS suggests to develop two different DSLs: one for the warehouse automation system logical view and one for the warehouse automation system physical view.

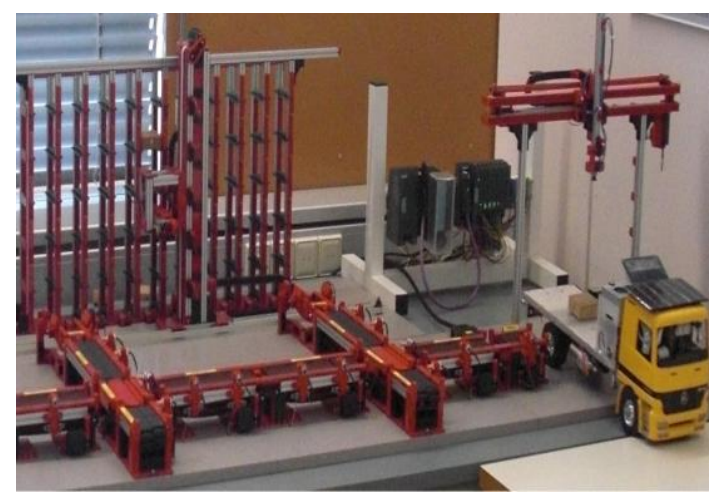

Fig. 1. Warehouse automation system-hardware prototype.

TABLE IV: DEVELOPMENT TIMES FOR THE TwO CASE STUDIES

\begin{tabular}{|l|c|c|}
\cline { 2 - 3 } \multicolumn{1}{l|}{} & WASPL-1 & WASPL-2 \\
\hline $\begin{array}{l}\text { Automation software development } \\
\text { time }[\mathrm{h}]\end{array}$ & 450 & 213 \\
$\begin{array}{l}\mathrm{DSL}+\mathrm{codegenerator} \mathrm{development} \\
\text { time }[\mathrm{h}]\end{array}$ & 450 & 81 \\
$\begin{array}{l}\text { Automation software development time } \\
\text { DSL+codegenerator development time }\end{array}$ & $50 \%$ & $27.6 \%$ \\
\hline \hline
\end{tabular}

\section{B. Development Time}

Table IV shows the developments times for both case studies. The development times are divided into automation software development and DSL including code generator development. We can see that the development times highly differ. WASPL-1 has got much higher development times for the automation software and for the DSL. However, both students who worked on the projects had similar skill levels (computer science students, no previous experiences in automation system programming or in DSLs). Even if we assume that the student working on WASPL-2 was more skilled than the student working on WASPL-1, the high differences in the development times are still difficult to explain.

If we just consider the relative amount of development time between the DSL and the automation software, it is interesting to see that the WASPL-2 DSL required less than one third of the overall development time, where the
WASPL-1 DSL required half of the overall development time. This indicates that the DSL architecture for WASPL-2 is better suited than the architecture for WASPL-1.

\section{Domain Model Complexity}

To measure the domain model complexity we use the metrics from [3]. These metrics simply count the elements of the metamodel and distinguish between interface complexity, element complexity, and property complexity. The metrics tailored for the GOPPRR (Graph-Object-Property-PortRole-Relationship) meta-metamodel which is used by MetaEdit+ are shown in the following equations where $\boldsymbol{C}$ stands for the complexity and $\boldsymbol{n}$ is the number of items.

$$
\begin{aligned}
& C_{\text {interface }}=n_{\text {Relationships }}+n_{\text {Roles }}+n_{\text {Constraints }} \\
& C_{\text {element }}=n_{\text {Objects }}+n_{\text {Ports }} \\
& C_{\text {property }}=n_{\text {Properties }}
\end{aligned}
$$

TABLE V: Domain Model CompleXity of THE Two CASE STUdies

\begin{tabular}{|l|c|c|}
\cline { 2 - 3 } \multicolumn{1}{c|}{} & WASPL-1 & WASPL-2 \\
\hline Interface Complexity & $1+4+16=\mathbf{2 1}$ & $2+12+1=\mathbf{1 5}$ \\
Property Complexity & $9+8=\mathbf{1 7}$ & $12+8=\mathbf{2 0}$ \\
\hline Overall Complexity & $\mathbf{2 6}$ & $\mathbf{1 1}$ \\
\hline \hline
\end{tabular}

TABLE VI: NUMBER OF ELEMENTS IN THE DSL MODELS

\begin{tabular}{|c|c|c|c|c|}
\hline Metric & Description & $\begin{array}{l}\text { Obtained } \\
\text { from }\end{array}$ & $\begin{array}{c}\text { WASPL- } \\
1\end{array}$ & $\begin{array}{c}\text { WASPL } \\
-2\end{array}$ \\
\hline$n_{l}$ & $\begin{array}{l}\text { Number of } \\
\text { distinct } \\
\text { operations }\end{array}$ & $\begin{array}{c}\text { number of Relationship } \\
\text { and Property types } \\
\text { (because they are actively } \\
\text { used to connect or refine } \\
\text { MetaEdit+ entities) }\end{array}$ & 27 & 13 \\
\hline$n_{2}$ & $\begin{array}{l}\text { Number of } \\
\text { distinct } \\
\text { operands }\end{array}$ & $\begin{array}{l}\text { number of Object types } \\
\text { (because they are } \\
\text { MetaEdit+ entities which } \\
\text { are first instantiated in the } \\
\text { model) }\end{array}$ & 9 & 12 \\
\hline$n$ & $n=n_{1}+n_{2}$ & & 36 & 25 \\
\hline$N_{l}$ & $\begin{array}{c}\text { Total number } \\
\text { of operators }\end{array}$ & $\begin{array}{l}\text { amount of Relationships } \\
\text { and Properties in a } \\
\text { specific model }\end{array}$ & 172 & 154 \\
\hline$N_{2}$ & $\begin{array}{c}\text { Total number } \\
\text { of operands }\end{array}$ & $\begin{array}{l}\text { amount of Objects in a } \\
\text { specific model }\end{array}$ & 16 & 26 \\
\hline$N$ & $N=N_{1}+N_{2}$ & & 188 & 180 \\
\hline$V$ & $\begin{array}{c}\text { Volume } \\
V=N^{*} \operatorname{ld}(n)\end{array}$ & & 972 & 836 \\
\hline$D$ & \begin{tabular}{|c|} 
Difficulty \\
$D=\left(n_{1} / 2\right) *\left(N_{2} /\right.$ \\
$\left.n_{2}\right)$
\end{tabular} & & 24 & 14 \\
\hline E & $\begin{array}{c}\text { Halstead } \\
\text { Effort } E=D^{*} V\end{array}$ & & 23,328 & 11,704 \\
\hline
\end{tabular}

\begin{tabular}{|l|c|c|}
\cline { 2 - 3 } \multicolumn{1}{c|}{} & WASPL-1 & WASPL-2 \\
\hline \#Objects & 16 & 26 \\
\#Relationship & 16 & 110 \\
A & 156 & 44 \\
\hline \#roperties & $\mathbf{1 8 8}$ & $\mathbf{1 8 0}$ \\
\hline \hline
\end{tabular}

TABLE VII: HALSTEAD EFFORT METRICS 
Table V shows the domain model complexity for the two case studies. We can see that WASPL-2 has a lower complexity. This directly confirms the MADMAPS approach which in our case suggests to split the domain model up into the logical and physical view (as it is done by WASPL-2) to obtain a more efficient domain model representation. It is interesting to see that WASPL-1 has a much higher property complexity. This is not surprising, because the hardware mapping of the automation system is modeled as properties of automation devices. In WASPL-2 the hardware mapping is modeled as additional objects and their connections. That is why the element complexity of WASPL-2 is higher.

\section{Model Size}

To compare how much effort it is to construct a model with the DSLs, we count the elements required in both DSL for modeling the system shown in Fig. 1 (see Table VI). We just counted the elements in MetaEdit+ which have to be manually added or edited for a MetaEdit+ model. For example, we did not count Ports and Roles, because they are implicitly added to a model by adding Relationships and Objects. We also counted the Properties, because they have to be manually set to configure DSL elements like Objects. We can see that both models nearly use the same amount of MetaEdit+ entities. This indicates that the required effort to construct a model is similar for both DSLs.

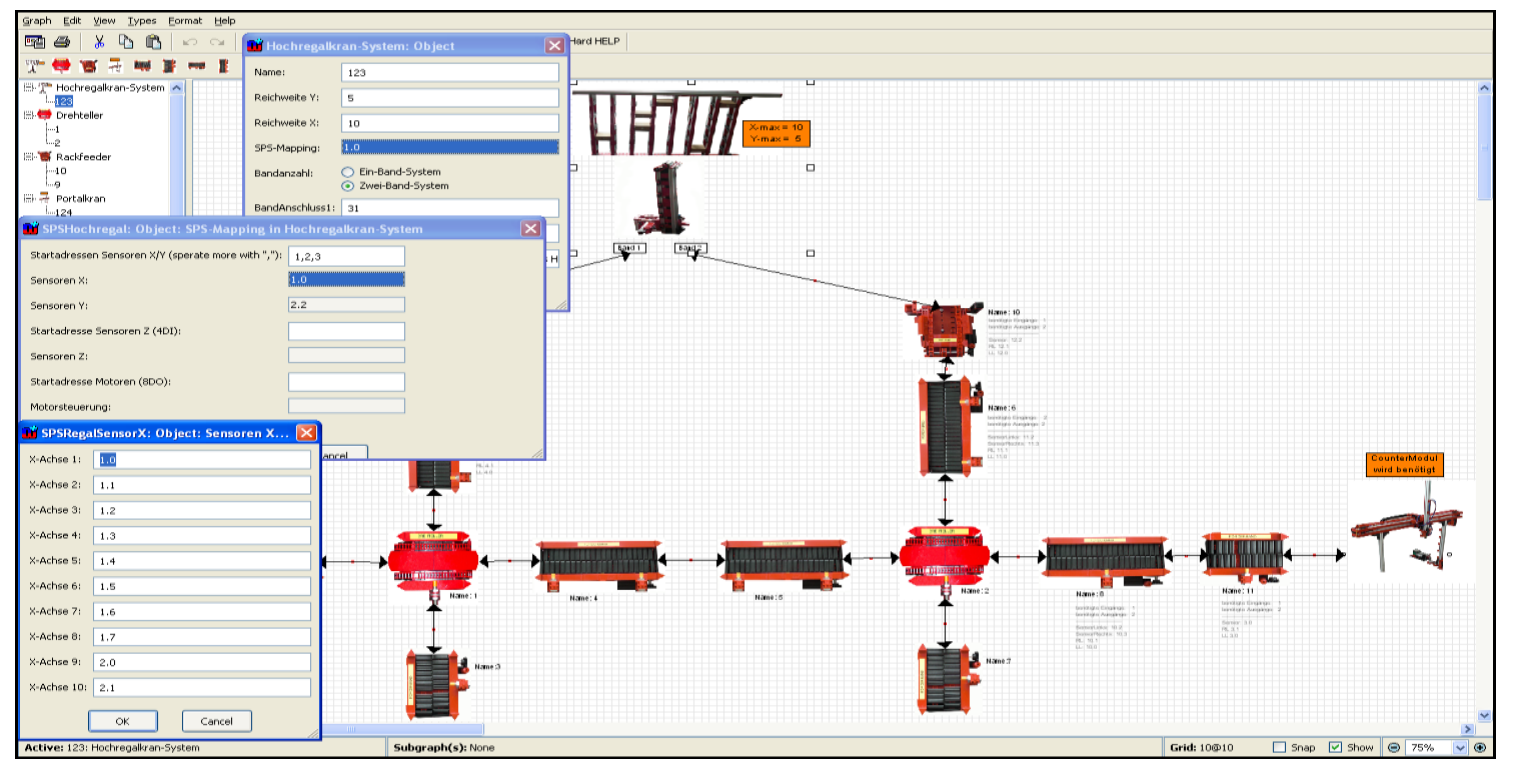

Fig. 2. WASPL-1 warehouse automation system model.

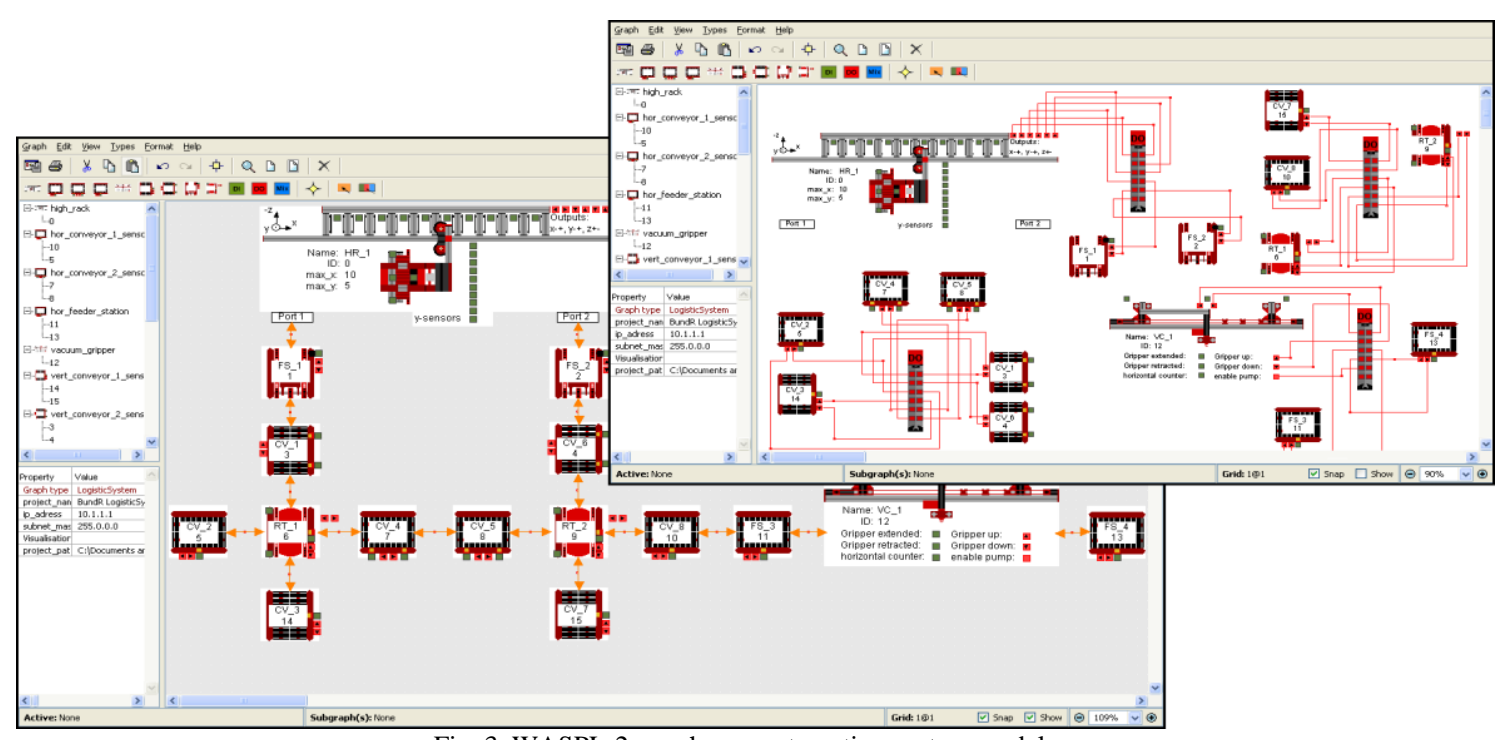

Fig. 3. WASPL-2 warehouse automation system model.

\section{E. Halstead Effort}

The Halstead effort [4] is a measure to evaluate how much effort it requires to develop or maintain a program. This measure is already applied to DSLs in [5]. The Halstead effort consists of the metrics shown in Table VII. In this table we also show how we obtain the metrics from the MetaEdit+ DSLs.

The right columns of Table VII show the calculated
Halstead effort for the two case studies. We can see that the Volume $\boldsymbol{V}$ of both DSL models is very similar. This indicates that both DSLs have the same level of abstraction or the same expressiveness to model the given system. However, we can see that the Difficulty $\boldsymbol{D}$ is much higher for WASPL-1. This is the case, because WASPL-1 provides so many different operators (e.g. Properties) which can refine DSL elements. According to the metrics, this makes understanding WASPL-1 more difficult. This statement also corresponds to 
our own experience with the two case studies. WASPL-1 was much more difficult to understand, because one had to understand all the exact Properties which had to be adjusted to configure the hardware mapping of the warehouse automation elements. In WASPL-2, this mapping was more intuitive, because for the hardware mapping, simply the wires of the automation system were graphically modeled. This resulted in a lower number of operators (Properties or Relationships) which can be applied to the automation elements. Due to the low Difficulty $\boldsymbol{D}$ for WASPL-2, this DSL has a much lower Halstead Effort $\boldsymbol{H}$.

\section{RELATED WORK}

This section presents related work on DSL evaluation metrics.

An extensive study to compare different DSLs is shown in [5]. The paper compares different implementation approaches (compiler-like architecture, source-to-source transformation, ...) for DSLs by developing the same DSL with these different approaches. The DSLs are then compared with different code complexity metrics (including the Halstead effort), the lines of code of the resulting textual DSL models, the time it takes for a user to develop a model, and the lines of code of the DSL code generators.

The DSL domain model complexity metric which we applied in this paper is discussed in [1] where the metrics are covered more general to be applied to DSM or to FODM. The metrics are applied to four industrial case studies some of which are developed with both, a DSM representation and a FODM representation.

Complexity metrics for DSL metamodels are discussed in [6]. The metrics are based on the minimum number of elements which is required to form a valid DSL model and on the dependence of the elements on one another. For example, if the instantiation of a DSL element requires another element, then the complexity of the metamodel rises. Further model-driven engineering metrics are discussed in [7]. The metrics are based on the goal question metrics approach and are explained in general as well as specifically form the EMOF meta-metamodel.

\section{CONCLUSION}

In this paper we presented two DSL case studies covering the same domain. We evaluated the case studies with the MADMAPS approach and with several general and DSL-related metrics.

The main difference between the two case studies was identified as their domain model structure, which is a compound domain model for one case study and a split domain model (split into the physical and logical view) for the other case study. The MADMAPS approach suggests that for the warehouse automation domain in the case studies, a split representation is better suited. The DSL evaluation shows that the DSL with split domain model achieved better results in terms of development effort, domain model complexity, and DSL difficulty/understandability (Halstead effort).
These results indicate that the MADMAPS suggestion to split up the domain model is a good early design decision. Of course one cannot clearly conclude that the evaluation metrics which we calculate for the DSLs clearly confirm the MADMAPS design decision; however, as all the measured metrics show better results for the DSL with the split domain model, these metrics suggest that the MADMAPS domain model recommendations for the warehouse automation domain are very good.

With this paper we confirmed the MADMAPS approach by applying it to two case studies and we hope to advertise the use of structured approaches for early stage domain modeling design decisions.

\section{REFERENCES}

[1] A. Leitner and C. Kreiner, "MADMAPS - Simple and systematic assessment of modeling concepts based on the nature of the domain," in Proc. EuroSPI Conference - European Systems \& Software Process Improvement and Innovation, Vienna, Austria, 2012, pp. 1-20.

[2] D. Winterfeldt and G. W. Fischer, "Multi-attribute utility theory: Models and assessment procedures," Utility, Probability, and Human Decision Making, ser. Theory and Decision Library, Springer Netherlands, 1975, vol. 11, pp. 47-85.

[3] A. Leitner and R. Wei, and C. Kreiner, "Analyzing the Complexity of Domain Model Representations," in Proc. 19th International Conference and Workshops on Engineering of Computer-Based Systems, Novi Sad, Serbia, IEEE, 2012, pp. 242-248.

[4] M. Halstead, Elements of Software Science, Elsevier North, 1977.

[5] T. Kosar, P. E. M. Lopez, P. A. Barrientos, M. Mernik, "A preliminary study on various implementation approaches of domain-specific language," Information and Software Technology, vol. 50, no. 5, 2007 pp. 390-405

[6] J. Sprinkle, "Analysis of a metamodel to estimate complexity of using a domain-specific language," in Proc. the 10th Workshop on Domain-Specific Modeling, Reno, USA, ACM, 2010, pp. 13:1-13:6.

[7] M. Monperrus, J.-M. Jezequel, J. Champeau, B. Hoeltzener, "Measuring models," Model-Driven Software Development: Integrating Quality Assurance. IDEA Group, 2008.

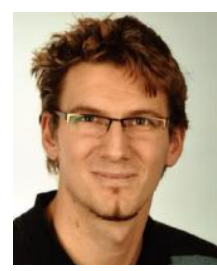

Christopher Preschern received the master degree in telematics from Graz University of Technology in 2011, focusing on software product lines, automation systems and IT-security. He is working toward the Ph.D. degree in electrical engineering at the Institute for Technical Informatics, Graz University of Technology. His research focuses on design patterns and their relationship to non-functional quality attributes, in particular safety and security.

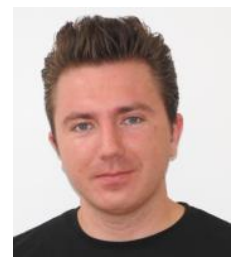

Nermin Kajtazovic received his master degree in telematics form Graz University of Technology in 2011. He focused on variability management in component-based architectures for the automotive domain. $\mathrm{He}$ is a $\mathrm{PhD}$ student at the Institute for Technical Informatics, Graz University of Technology. His research is related to component-based systems in the safety domain.

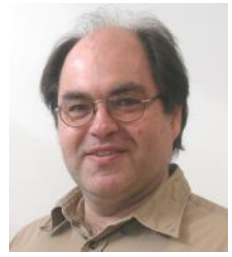

Christian Kreiner graduated and received the Ph.D. degree in electrical engineering from Graz University of Technology, in 1991 and 1999, respectively. From 1999 to 2007, he served as head of the R\&D Department, Salomon Automation, Austria, focusing on software architecture, technologies, and processes for logistics software systems. He was in charge to establish a company-wide software product line development process and headed the product development team. There, he led and coordinated a long-term research program together with the Institute for Technical Informatics of Graz University of Technology. There, he currently leads the Industrial Informatics and Model-based Architectures group. His research interests include systems and software engineering, software technology, and process improvement. 